\title{
Severe MRI-visible perivascular spaces due to cerebral amyloid angiopathy
}

\author{
Sayan Datta, ${ }^{1}$ Hawraman Ramadan, ${ }^{1}$ David J Werring ${ }^{2}$
}

\begin{abstract}
${ }^{1}$ Department of Neurology, Bradford Royal Infirmary, Bradford, UK

${ }^{2}$ Stroke Research Group, Department of Brain Repair and Rehabilitation, UCL Institute of Neurology, London WC1N 3BG, UK
\end{abstract}

\section{Correspondence to} Dr Hawraman Ramadan, Department of Neurology, Bradford Teaching Hospitals NHS Trust, Duckworth lane, Bradford Bd6 6 RJ, UK; Hawraman. Ramadan@bthft.nhs.uk

Accepted 2 September 2014 Published Online First 26 September 2014

\section{CrossMark}

\footnotetext{
To cite: Datta S, Ramadan $\mathrm{H}$, Werring DJ. Pract Neurol 2015;15:74-75.
}

\section{SUMMARY}

MRI-visible perivascular spaces are a recently recognised neuroimaging marker of cerebral small vessel diseases ${ }^{1}$ and centrum semiovale MRI-visible perivascular spaces may be a neuroimaging marker of cerebral amyloid angiopathy. ${ }^{2}$

We report an elderly woman with speech and memory disturbance, whose MR scan of brain showed severe centrum semiovale perivascular spaces. Subsequent MRI with blood-sensitive sequences showed multiple cerebral microbleeds, consistent with probable cerebral amyloid angiopathy. Dilated perivascular spaces can be a marker of cerebral amyloid angiopathy. Their recognition may change clinical management, since current recommendations for cerebral amyloid angiopathy treatment involve avoiding antithrombotic drugs and emphasising hypertensive control.

\section{CASE REPORT}

A previously healthy 81-year-old woman gave a 3-month history of intermittent word-finding difficulty and prosopagnosia. Her short-term memory was normal and she was independent with activities of daily living. Her neurological examination was normal.

Her initial MR scan of brain showed extensive severe MRI-visible perivascular spaces on T2-weighted sequences (figure $1 \mathrm{~A}-\mathrm{C}$ ) but was otherwise normal. Further imaging with susceptibility-weighted imaging showed multiple strictly lobar cerebral microbleeds and focal cortical superficial siderosis (figure 1D-F), consistent with cerebral amyloid angiopathy.

\section{COMMENT}

This patient fulfils the modified Boston criteria for probable cerebral amyloid angiopathy: age $\geq 55$ years, with multiple haemorrhages restricted to lobar, cortical or subcortical brain regions (cerebellar allowed), focal or disseminated superficial siderosis and no other identified cause of cerebral haemorrhage.

The brain's perivascular spaces are a normal elimination pathway for interstitial fluid and toxic solutes, including amyloid-beta. Dilatation of perivascular spaces on MRI is now recognised as being associated with small vessel disease; associated factors include ageing, diabetes mellitus, hypercholesterolaemia, smoking and hypertension.

Dilatation of the perivascular spaces in the cerebral white matter in cerebral amyloid angiopathy may relate to impaired interstitial fluid drainage due to amyloid deposition in superficial cortical and leptomeningeal or peforating white matter arterioles. $^{2}$ Their detection on MR scanning may be a promising new marker of cerebral amyloid angiopathy. Hypertensive vasculopathy is more strongly associated with basal ganglia perivascular spaces, whereas in cerebral amyloid angiopathy they are more distributed in the hemisphere white matter. ${ }^{2}$

Cerebral amyloid angiopathy is an important cause of cognitive decline; clinicians thus need a high index of suspicion in patients with unexplained cognitive impairment. Appropriate blood-sensitive MRI sequences (e.g. gradient echo T2* or susceptibility weighted imaging) should become routine in elderly patients with cognitive impairment, since they can reveal cerebral microbleeds and cortical superficial siderosis as low-signal abnormalities.

In this case, centrum semiovale perivascular spaces prompted us to request further MR scans with blood-sensitive MRI sequences. The imaging findings changed her clinical management, since secondary prevention in cerebral amyloid angiopathy involves antihypertensives and avoidance of antithrombotic medications; disease-modifying treatments including a 

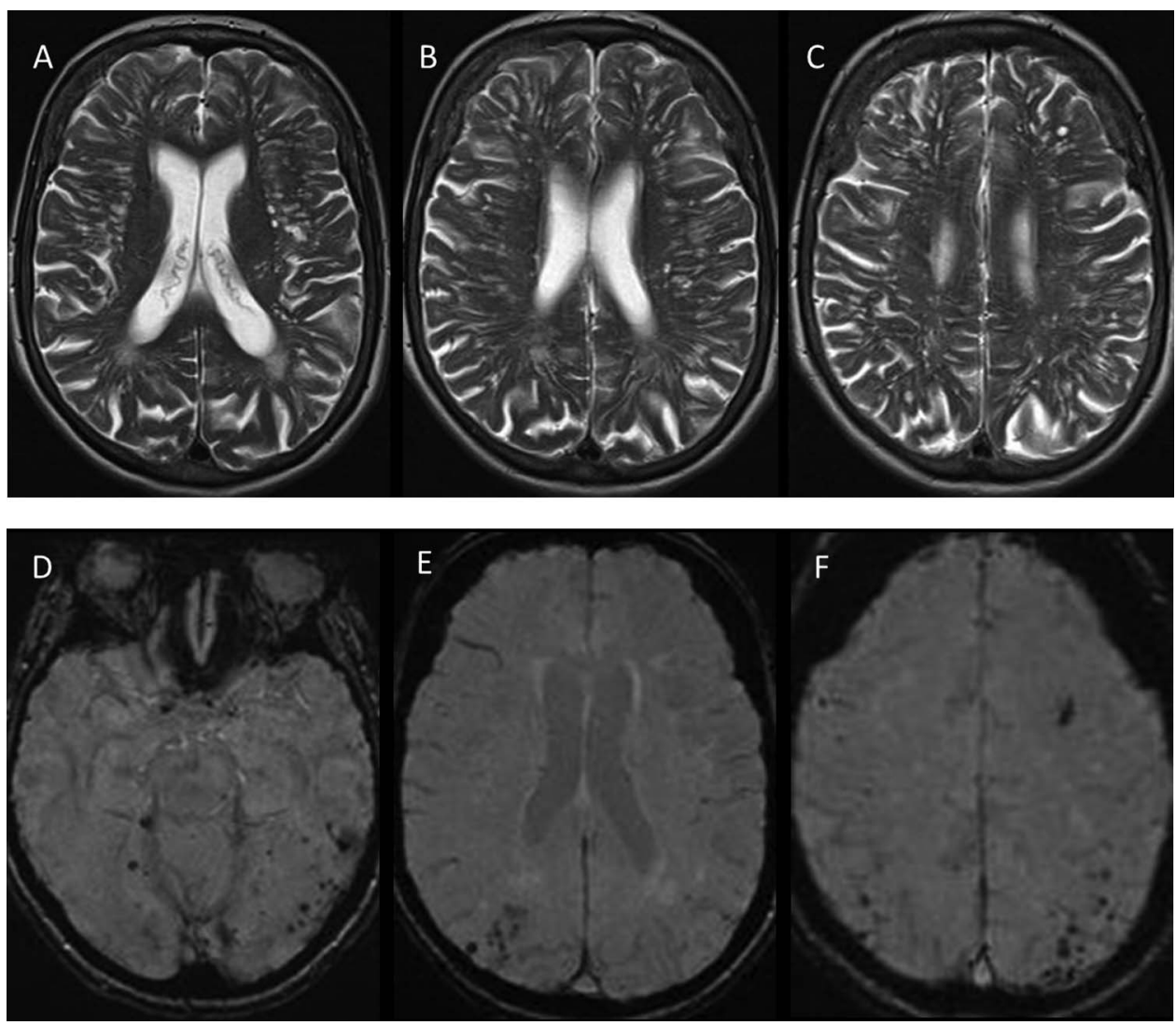

Figure 1 (A-C) MR scan of brain (T2-weighted sequence) showing severe centrum semiovale dilated perivascular spaces. (D-F) MR scan of brain (susceptibility-weighted imaging) showing multiple lobar cerebral microbleeds and focal superficial siderosis.

monoclonal antibody targeting vascular amyloid are also under investigation (see: http://clinicaltrials.gov/ ct2/show/NCT01821118).

Contributors SD drafted the article and coordinated subsequent revisions. HR gave the concept for the article and revised the original draft. DW provided expert opinion with further revisions to the article.

\section{Competing interests None.}

Patient consent Obtained.
Provenance and peer review Not commissioned; externally peer reviewed. This paper was reviewed by Maurice Moriarty, Auckland, New Zealand.

\section{REFERENCES}

1 Wardlaw J, Smith E, Biessels G, et al. Neuroimaging standards for research into small vessel disease and its contribution to ageing and neurodegeneration. Lancet Neurol 2013;12:822-38.

2 Charidimou A, Jaunmuktane Z, Baron JC, et al. White matter perivascular spaces: An MRI marker in pathology-proven cerebral amyloid angiopathy? Neurology 2014;82:57-62. 